\title{
The Dynamics of Lysozyme from Bacteriophage Lambda in Solution Probed by NMR and MD Simulations
}

\author{
Lorna J. Smith, ${ }^{*[a]}$ Alice M. Bowen, ${ }^{[a]}$ Alexandre Di Paolo, ${ }^{[b]}$ André Matagne, ${ }^{[b]}$ and \\ Christina Redfield ${ }^{*[c]}$
}

In memory of Ivano Bertini's enthusiastic contributions to biomolecular science.

\begin{abstract}
${ }^{15} \mathrm{~N}$ NMR relaxation studies, analyses of NMR data to include chemical shifts, residual dipolar couplings (RDC), NOEs and $\mathrm{H}^{\mathrm{N}}-$ $\mathrm{H}^{\alpha}$ coupling constants, and molecular dynamics (MD) simulations have been used to characterise the behaviour of lysozyme from bacteriophage lambda ( $\lambda$ lysozyme) in solution. The lower and upper lip regions in $\lambda$ lysozyme (residues $51-60$ and $128-141$, respectively) show reduced ${ }^{1} \mathrm{H}-{ }^{15} \mathrm{~N}$ order parameters indicating mobility on a picosecond timescale. In addition, residues in the lower and upper lips also show exchange contributions to $T_{2}$ indicative of slower timescale motions. The chemical
\end{abstract}

shift, RDC, coupling constant and NOE data for $\lambda$ lysozyme indicate that two fluctuating $\beta$-strands ( $\beta 3$ and $\beta 4)$ are populated in the lower lip region while the $N$ terminus of helix $\alpha 6$ (residues 136-139) forms dynamic helical turns in the upper lip region. This behaviour is confirmed by MD simulations that show hydrogen bonds, indicative of the $\beta$-sheet and helical secondary structure in the lip regions, with populations of 40 $60 \%$. Thus in solution $\lambda$ lysozyme adopts a conformational ensemble that will contain both the open and closed forms observed in the crystal structures of the protein.

\section{Introduction}

Lysozyme from bacteriophage lambda ( $\lambda$ lysozyme) is a monomeric 158 residue enzyme that catalyses the cleavage of the glycosidic bonds in bacterial peptidoglycan between $\mathrm{N}$-acetylmuramic acid (MurNAc) and $\mathrm{N}$-acetylglucosamine (GlcNAc) residues. ${ }^{[1]}$ Unlike most lysozymes, which catalyse hydrolysis, the cleavage mechanism for $\lambda$ lysozyme involves intramolecular transglycosylation forming a 1,6-anhydro- $N$-acetylmuramic acid terminal residue. ${ }^{[2]}$ In this respect $\lambda$ lysozyme resembles the lytic transglycosylases. ${ }^{[3]}$

Similar to all lysozymes, the structure of $\lambda$ lysozyme consists of two domains (Figure 1). ${ }^{[4]}$ Domain I contains two $\beta$-sheets formed from six $\beta$-strands and one $\alpha$-helix ( $\alpha 2)$, while domain II contains four $\alpha$-helices $(\alpha 1, \alpha 4, \alpha 5, \alpha 6)$. A sixth $\alpha$-helix $(\alpha 3)$ links the two domains. The active-site cleft in $\lambda$ lysozyme lies between the two domains. The essential catalytic residue, Glu19, is positioned deep within this cleft. ${ }^{[5]}$ Comparison of the structure of $\lambda$ lysozyme with that of c-type and v-type lysozymes has shown some similarities. ${ }^{[4]]}$ Structural alignment of about $60 \mathrm{C}^{\alpha}$ atoms in $\lambda$ lysozyme with T4 lysozyme and hen

[a] Dr. L. J. Smith, Dr. A. M. Bowen

Department of Chemistry, University of Oxford

Inorganic Chemistry Laboratory

South Parks Road, Oxford OX1 3QR (UK)

E-mail: lorna.smith@chem.ox.ac.uk

[b] Dr. A. Di Paolo, Prof. Dr. A. Matagne

Laboratoire d'Enzymologie et Repliement des Protéines

Centre d'Ingénierie des Protéines, Institut de Chimie B6, Université de Liège

Allée de la Chimie 3, 4000 Liège, Sart-Tilman (Belgium)

[c] Prof. Dr. C. Redfield

Department of Biochemistry, University of Oxford

South Parks Road, Oxford OX1 3QU (UK)

E-mail:christina.redfield@bioch.ox.ac.uk egg-white lysozyme, including particularly helices $\alpha 1$ and $\alpha 3$ and part of the $\beta$-sheets in $\lambda$ lysozyme, gives an atom positional RMSD of approximately $2 \AA .^{[4 a]}$ In addition, there is a similarity between the structure of $\lambda$ lysozyme and the peptidoglycan glycosyltransferase (PGT) domains from Staphylococcus aureus $^{[6]}$ and Aquifex aeolicus ${ }^{[7]}$ penicillin-binding proteins. Here there is good overlay of secondary structure between domain I of $\lambda$ lysozyme and the larger globular "head" lobe of the PGT domain, including helices $\alpha 1, \alpha 3, \alpha 5$ and $\alpha 6$ in $\lambda$ lysozyme. Glu19, in the active site of $\lambda$ lysozyme, also overlays with a catalytic glutamate residue in PGT. However, domain II in $\lambda$ lysozyme is replaced by a membrane associated helical "jaw" lobe in the PGT domains. ${ }^{[6,7]}$

One interesting feature from the crystallographic studies of $\lambda$ lysozyme is the observation of two different conformations, open and closed forms, in the region surrounding the enzyme active site. ${ }^{[4]}$ In particular, the X-ray structure with no inhibitor ligand bound in the active site (PDB ID: $1 A M 7^{[4 a]}$ ) contains three molecules in the asymmetric unit, molecules $A$ and $C$ adopting the open form and molecule $B$ the closed form. The closed form is also seen in the $\mathrm{X}$-ray structure of $\lambda$ lysozyme in complex with hexa- $N$-acetylchitohexaose (PDB ID: 3D3D). ${ }^{[4 b]}$ The significant differences between the open and closed forms are for residues 51-60 (lower lip) and 128-141 (upper lip). ${ }^{[4]}$ The lower lip contains two $\beta$-strands ( $\beta 3$ and $\beta 4$ ) in the open form. These $\beta$-strands are missing in the closed form, the lower lip forming a long loop which partially restricts access to the active-site cleft. In the upper lip region the main differences between the open and closed forms are at the $\mathrm{N}$ terminus of helix $\alpha 6$. In the open form helix $\alpha 6$ comprises residues 135148 but in the closed form the first helical turn is unwound 


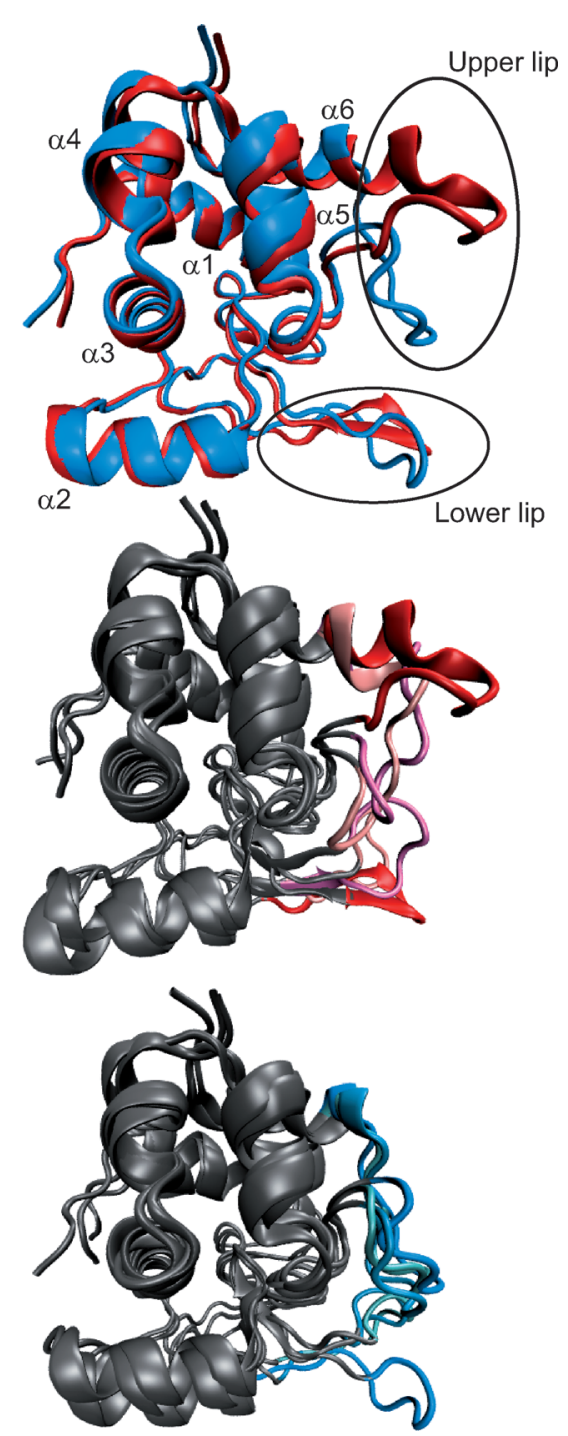

Figure 1. Upper panel: Ribbon representation of molecules A (red; open form) and B (blue; closed form) in the X-ray structure of $\lambda$ lysozyme with PDB ID: $1 \mathrm{AM7} 7{ }^{[4 a]}$ The lower and upper lip regions (residues 51-60 and 128141 , respectively), which show different conformations, are indicated. The six $\alpha$-helices in the protein are labelled. Middle and lower panels: Superposition of instantaneous structures taken from the MD simulations of $\lambda$ lysozyme. The protein backbone is shown in ribbon representation in grey with the lower and upper lip regions shown in colour. Middle panel: simulation of molecule A: red $0 \mathrm{~ns}$, mauve $5 \mathrm{~ns}$, pink $10 \mathrm{~ns}$ structures. Lower panel: simulation of molecule B: blue 0 ns, light blue 5 ns, cyan 10 ns structures.

(helix $\alpha 6$ comprises residues 139-148) and the upper loop region extends across the active site. ${ }^{[4]}$

In this paper we report the characterisation of the structure and dynamics of $\lambda$ lysozyme in solution by NMR techniques, including ${ }^{15} \mathrm{~N}$ relaxation studies, ${ }^{15} \mathrm{~N}-{ }^{1} \mathrm{H}^{\mathrm{N}}$ residual dipolar coupling measurements and analyses of chemical shift, NOE, coupling constant and hydrogen exchange data. The experimental data are complemented by two molecular dynamics (MD) simulations starting from the open and closed conformations seen in the X-ray structures. We concentrate particularly on the behaviour of the upper and lower lip regions, both of which show considerable conformational flexibility in solution.

\section{Results and Discussion}

\section{${ }^{15} \mathrm{~N}$ relaxation measurements}

${ }^{15} \mathrm{~N}$ relaxation measurements $\left(T_{1}, T_{2}\right.$ and $\left\{{ }^{1} \mathrm{H}\right\}^{15} \mathrm{~N}$ heteronuclear NOE) have been carried out for $\lambda$ lysozyme at 500 and $750 \mathrm{MHz}$; data were obtained for 128 of the 158 residues of $\lambda$ lysozyme. The $T_{1} / T_{2}$ ratios at 500 and $750 \mathrm{MHz}$ are shown in Figure $2 \mathrm{~A}$. As expected, ${ }^{15} \mathrm{~N} T_{1}$ values increase with frequency
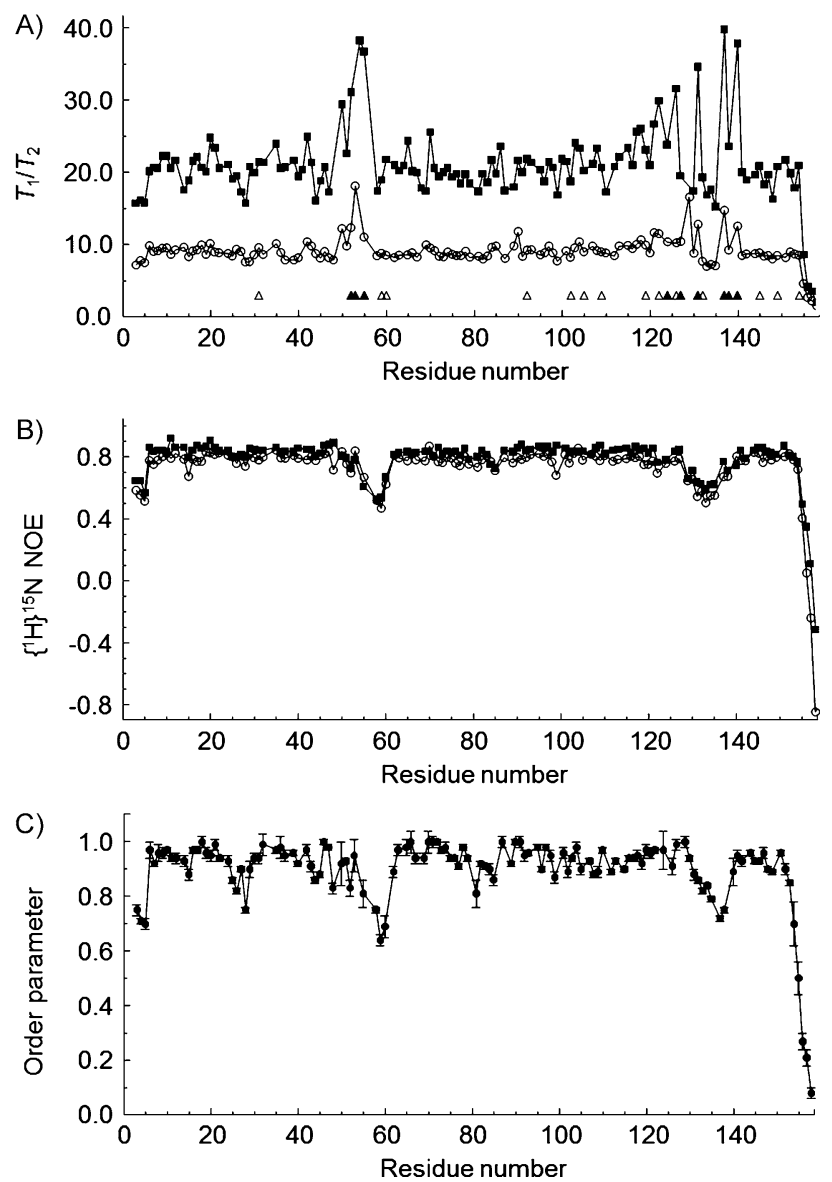

Figure 2. A) Experimental $T_{1} / T_{2}$ ratios obtained at $500 \mathrm{MHz}(O)$ and at $750 \mathrm{MHz}(\mathbf{\square})$. Residues requiring an exchange contribution $\left(R_{\mathrm{ex}}\right)$ to $T_{2}$ are indicated by $\triangle$ for values less than $1.0 \mathrm{~s}^{-1}$, and by $\boldsymbol{\Delta}$ for values greater than $1.0 \mathrm{~s}^{-1}$, just above the $x$-axis. For all residues $R_{\mathrm{ex}}$ values are given for $500 \mathrm{MHz}$ and must be scaled by 2.25 for $750 \mathrm{MHz}$. B) Experimental $\left\{{ }^{1} \mathrm{H}\right\}^{15} \mathrm{~N}$ heteronuclear NOE ratios obtained at $500 \mathrm{MHz}(\mathrm{O})$ and at $750 \mathrm{MHz}(\mathbf{\square})$. C) $S^{2}$ order parameters obtained from the fitting of the ${ }^{15} \mathrm{~N}$ relaxation data using the "model-free" approach, as described in the Experimental Section. Errors derived from the Monte Carlo analysis are shown.

while $T_{2}$ values generally decrease. Some residues have $T_{2}$ values that show more complex frequency dependencies. It is clear from the $T_{1} / T_{2}$ ratios, particularly at $750 \mathrm{MHz}$, that exchange contributions to $T_{2}$ are observed for some residues, particularly in the region of residues $51-60$ and 124-140. $\left\{{ }^{1} \mathrm{H}\right\}^{15} \mathrm{~N}$ NOE ratios determined at 500 and $750 \mathrm{MHz}$ are shown in Figure 2B. For the majority of residues, NOE ratios above 0.75 are observed, indicating a rigid backbone, characteristic of a globular protein with limited amplitude dynamics on a fast 
timescale. However, lower NOE ratios, indicative of significant dynamics on the picosecond timescale are observed in several regions of the sequence, including residues at the $\mathrm{N}$ and $\mathrm{C}$ termini but also for residues 52-60 and 129-138. These latter two groups of residues correspond to the two regions found in different conformations in the open and closed structures identified by X-ray crystallography. ${ }^{[4]}$

The ${ }^{15} \mathrm{~N} T_{1}, T_{2}$ and $\left\{{ }^{1} \mathrm{H}\right\}^{15} \mathrm{~N}$ heteronuclear NOE data were analysed using the "model-free" approach of Lipari and Szabo, ${ }^{[8]}$ using an axially symmetric diffusion tensor, as described in the Experimental Section. Six different models were tested; the fitted parameters in these models include $S^{2}$, the generalized order parameter, $R_{\mathrm{ex}}$ the chemical exchange contribution to $T_{2^{\prime}}$ $\tau_{\mathrm{e}}$, the effective correlation time for internal motion on a fast timescale, $S^{2}{ }_{f} S^{2}$ and $\tau_{s}$ the order parameters for faster and slower timescale motions and the effective correlation time for slow internal motion. Model $1\left(S^{2}\right.$ only) was selected for 86 of the 128 residues analysed, 12 residues required $S^{2}$ and $R_{\mathrm{ex}}$ (model 2), six required $S^{2}$ and $\tau_{\mathrm{e}}$ (model 3 ), six required $S^{2}, R_{\mathrm{ex}}$ and $\tau_{\mathrm{e}}$ (model 4 ), 13 residues required the use of the extended model with $S^{2}{ }_{s} S^{2}{ }_{f}$ and $\tau_{s}$ (model 5) and five residues required the use of the extended model $\left(S^{2}{ }_{s} S^{2}{ }_{f}\right.$ and $\left.\tau_{s}\right)$ with $R_{\text {ex }}$ (model 6). The order parameters $\left(S^{2}\right)$ obtained from the modelfree analysis are plotted as a function of sequence in Figure $2 C$. Generally high $S^{2}$ values $(\geq 0.85)$ are obtained for the majority of residues ( $83 \%)$ in $\lambda$ lysozyme indicating a rigid backbone in most regions of the structure. However, lower order parameters $(<0.85)$ are observed for 22 residues; these are located at the $\mathrm{N}$ and $\mathrm{C}$ termini of the sequence and also for residues 55-60 and 133-138, these residues correspond to the lower and upper lip regions found to adopt different conformations in the $1 \mathrm{AM} 7$ crystal structure. ${ }^{[4 a]}$ Chemical exchange contributions to $T_{2}, R_{\text {ex }}$ terms, were required for 23 residues as shown in Figure $2 \mathrm{~A}$. It is interesting to note that the residues with the largest $R_{\text {ex }}$ terms are located in the lower and upper lip regions indicating that these residues undergo motions on both fast picosecond and slower microsecond to millisecond timescales.

\section{Chemical shift, coupling con- stant and NOE data for the lip regions}

The ${ }^{1} \mathrm{H},{ }^{13} \mathrm{C}$, and ${ }^{15} \mathrm{~N}$ chemical shifts for $\lambda$ lysozyme ${ }^{[9]}$ have been compared with those predicted using the SHIFTX2 ${ }^{[10]}$ program for molecules $A$ and $B$ in the $1 \mathrm{AM} 7$ crystal structure. ${ }^{[4 a]}$ TALOS $+{ }^{[11]}$ has also been used to predict the $\phi, \psi$ torsion angles from the experimental chemical shift data and these torsion angles have been com- pared with those seen in the crystal structures. Overall, considering the full sequence of $\lambda$ lysozyme, there is a good agreement between the experimental chemical shifts and those predicted using SHIFTX2. For example, for the ${ }^{13} \mathrm{C}^{\alpha}$ chemical shifts there are correlation coefficients of 0.995 and 0.993 between the experimental chemical shifts and those calculated for molecules A (open form) and B (closed form), respectively, whereas for the main-chain ${ }^{15} \mathrm{~N}$ chemical shifts, the correlation coefficients are 0.977 and 0.914 , respectively.

In the lower lip region the experimental chemical shifts are very similar to those predicted by SHIFTX2 for molecule A (open form; Figure $3 \mathrm{~A}$ ). However, differences compared to the predictions for molecule $B$ (closed form) of at least $2 \mathrm{ppm}$ in $\mathrm{C}^{\alpha}$ chemical shift are seen for Thr54 and Lys60 and of at least $4 \mathrm{ppm}$ in main chain ${ }^{15} \mathrm{~N}$ chemical shift for Leu52, Val53, Thr54 and Leu59. With TALOS+ the $\phi, \psi$ torsion angles predicted from the experimental chemical shifts for the lower lip region agree well with those observed for molecule $A$ in the crystal structure but there are significant differences for the $\psi$ torsion angles of Val53 and Leu59 from the values seen for molecule B in the crystal structure. For Val53 TALOS+ predicts a $\beta$-sheet conformation with a $\phi$ angle of $(-97 \pm 19)^{\circ}$ and a $\psi$ angle of $+128 \pm 9^{\circ}$ while in molecules $A$ and $B$ in the crystal structure the $\phi, \psi$ torsion angles are $-102^{\circ},+132^{\circ}$ and $-119^{\circ},+2^{\circ}$, respectively. Similarly for Leu59 TALOS + predicts a $\beta$-sheet conformation with a $\phi$ angle of $-130 \pm 25^{\circ}$ and a $\psi$ angle of $+121 \pm 21^{\circ}$ while in molecules $A$ and $B$ in the crystal structure the $\phi, \psi$ torsion angles are $-123^{\circ},+109^{\circ}$ and $-92^{\circ},-6^{\circ}$, respectively.

${ }^{3} \mathrm{JNH}_{\mathrm{HN \alpha}}$ coupling constants have been measured for 104 residues in $\lambda$ lysozyme and have been compared with values predicted from the $\phi$ torsion angles in molecules $A$ and $B$ from the 1AM7 crystal structure. Overall, better agreement is seen between the experimental coupling constants and those pre-
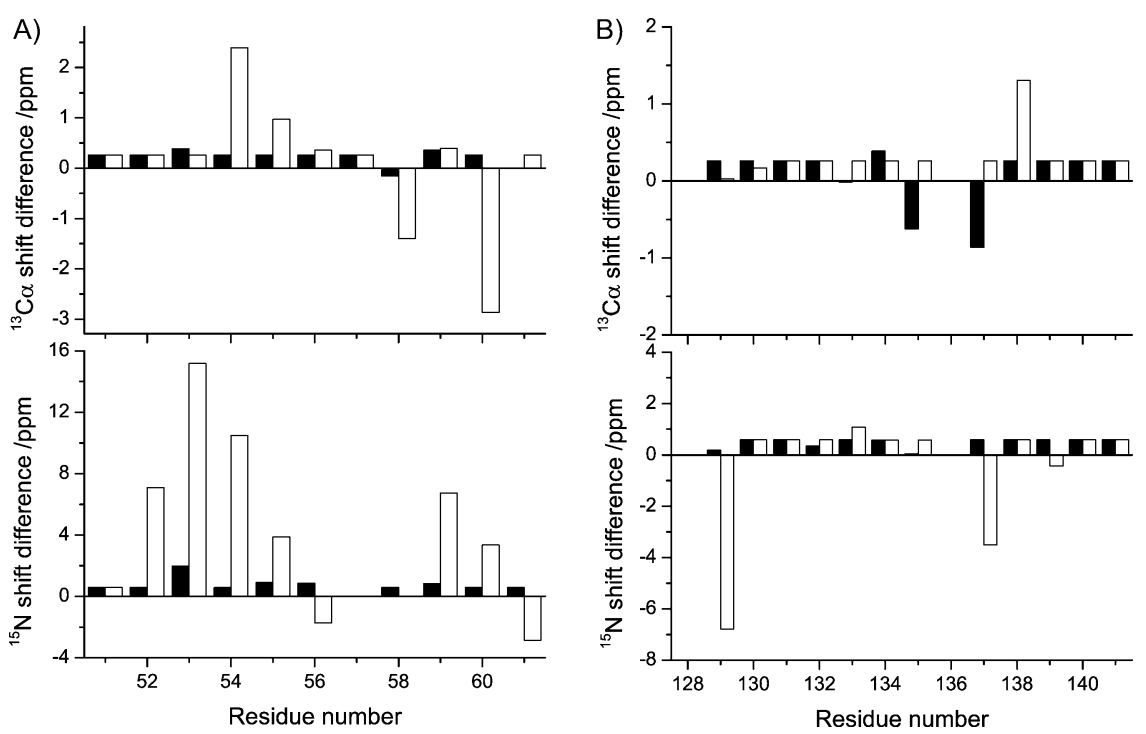

Figure 3. Difference between predicted and experimental ${ }^{13} \mathrm{C}^{\alpha}$ (upper panels) and ${ }^{15} \mathrm{~N}$ (lower panels) chemical shifts for A) residues 51-61 (lower lip) and B) 128-141 (upper lip) in $\lambda$ lysozyme. The program SHIFTX2 ${ }^{[10]}$ was used to predict the chemical shifts for molecules $A$ (open form) and B (closed form) in the crystal structure of $\lambda$ lysozyme with PDB ID: 1 AM7. ${ }^{[4]}$ The chemical shift difference (experiment-predicted) for molecules A and B are shown with filled bars and open bars, respectively. 
dicted from the structure of molecule A (correlation coefficient 0.860 ) compared to those predicted from the structure of molecule $B$ (correlation coefficient 0.788 ). In the lower lip region experimental ${ }^{3} J_{\mathrm{HNH} \alpha}$ coupling constant data are available for Lys51, Leu52, Thr54, Leu55, Lys58, Leu59 and Lys60. For three of these residues, Leu52, Lys58 and Lys60, there are significant differences greater than $2.5 \mathrm{~Hz}$ between the experimental coupling constants and those predicted from the structure of molecule B. Some differences are also seen for these residues from those predicted from the structure of molecule $A$, but in each case there is a closer agreement (Leu52 experimental $6.2 \mathrm{~Hz}$, predicted molecule A $8.3 \mathrm{~Hz}$, molecule B $9.6 \mathrm{~Hz}$; Lys 58 experimental $9.4 \mathrm{~Hz}$, predicted molecule $A 8.1 \mathrm{~Hz}$, molecule $B 6.8 \mathrm{~Hz}$; Lys60 experimental $8.6 \mathrm{~Hz}$, predicted molecule $A 9.7 \mathrm{~Hz}$, molecule B $3.1 \mathrm{~Hz}$ ).

In molecule $A$ (open form) there are $\beta$-strands for residues 53-56 ( $\beta 3$ ) and 59-61 ( $\beta 4)$ but both $\beta$-strands are missing in molecule B (closed form). The significantly better agreement seen for the chemical shift data for residues in these regions with the predictions for molecule A compared to molecule $B$ suggest that the $\beta$-strands are significantly populated in solution. This conclusion is also supported by NOE data. In particular, analysis of interproton distances in the 1AM7 X-ray structure indicates that long-range interstrand NOEs are expected between residues $54,55,56(\beta 3)$ and residues $59,60,61(\beta 4)$ in the open (molecule A) but not in the closed (molecule B) conformation. Experimentally long-range NOEs between $\mathrm{L} 55 \mathrm{H}^{\mathrm{N}}$, $\mathrm{N} 56 \mathrm{H}^{\mathrm{N}}$ and $\mathrm{K} 60 \mathrm{H}^{\alpha}$ and between $\mathrm{S} 61 \mathrm{H}^{\mathrm{N}}$ and $\mathrm{T} 54 \mathrm{H}^{\alpha}$ are observed (Figure 4). However, similar intensities are seen between $\mathrm{L} 55 \mathrm{H}^{\mathrm{N}}, \mathrm{N} 56 \mathrm{H}^{\mathrm{N}}$ and $\mathrm{K} 60 \mathrm{H}^{\alpha}$ while the open structure predicts distances of 2.9 and $4.5 \AA$, respectively. The observation of

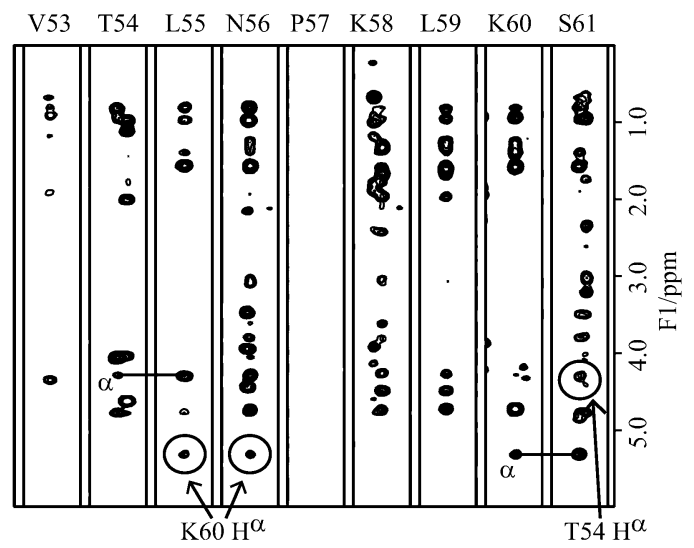

$\begin{array}{lllllllll}\text { K138 } & \text { A139 } & \text { D140 } & \text { S141 } & \text { L142 } & \text { I143 } & \text { A144 } & \text { K145 } & \text { F146 }\end{array}$

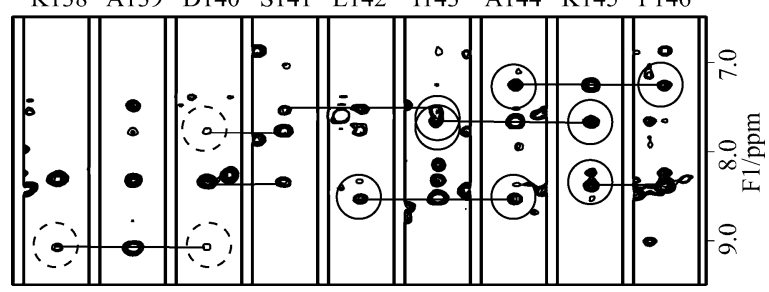

Figure 4. Upper panel: Strips from a $3 \mathrm{D}^{15} \mathrm{~N}$-edited NOESY-HSQC spectrum showing sequential and longer-range $\mathrm{H}^{\alpha}, \mathrm{H}^{\mathrm{N}}$ NOEs observed for residues 5361 in $\lambda$ lysozyme. Lower panel: Strips showing strong (full circles) and weak (dashed circles) sequential $\mathrm{H}^{\mathrm{N}}, \mathrm{H}^{\mathrm{N}}$ NOEs observed for residues $138-146$ in $\lambda$ lysozyme. these NOEs indicates that the $\beta 3$ and $\beta 4$ strands, as seen in the open conformation, must be populated in solution. However, the similar peak intensities suggest that there is some conformational averaging in this region. Conformational averaging may also be contributing to the differences seen in this region between experimental ${ }^{3} J_{\mathrm{HNH}}$ coupling constants and those predicted from the molecule $A$ structure. In addition, studies of hydrogen/deuterium (H/D) exchange in $\lambda$ lysozyme $^{[12]}$ show that there is no protection from H/D exchange for residues 53-61, indicating that the hydrogen bonds between the $\beta$-strands in this region must not be very persistent.

In the upper lip region residues 128-134 adopt a loop conformation with similar $\phi, \psi$ torsion angles in molecules $\mathrm{A}$ and B. Good agreement is seen between the SHIFTX2 predictions for both molecules $A$ and $B$ and the experimental data (Figure $3 \mathrm{~B}$ ), with the exception of Gly 129 where a deviation greater than $6 \mathrm{ppm}$ is seen for the main chain ${ }^{15} \mathrm{~N}$ prediction for molecule $B$. In molecule $A$ helix $\alpha 6$ starts at Phe135 whereas in molecule $B$ the helix does not start until Ala139. Using the experimental chemical shift data, TALOS + predicts a helix starting from Glu136 (there is an ambiguous $\phi, \psi$ prediction for Phe135). There are significant differences between the $\phi, \psi$ predictions from the chemical shift data for Glu136 ( $\phi-65 \pm$ $\left.3^{\circ}, \psi-40 \pm 5^{\circ}\right)$ and Lys138 $\left(\phi-66 \pm 5^{\circ}, \psi-40 \pm 4^{\circ}\right)$ and those observed in molecule B (Glu136 $\phi-121^{\circ}, \psi+131^{\circ}$; Lys138 $\phi$ $-79^{\circ}, \psi+152^{\circ}$ ) while the agreement with molecule $\mathrm{A}$ is much better (Glu136 $\phi-52^{\circ}, \psi-42^{\circ}$; Lys138 $\phi-68^{\circ}, \psi-39^{\circ}$ ). Thus the chemical shift data predict that the final residues of the upper lip region adopt a helical conformation as seen in the open conformation.

Analyses of interproton distances in molecules $A$ and $B$ in the $1 A M 7$ crystal structure predict that sequential $\mathrm{H}^{\mathrm{N}}-\mathrm{H}^{\mathrm{N}}$ NOEs should be seen for residues 137-146 in the upper lip region. However, in the open conformation $\mathrm{H}^{\mathrm{N}}-\mathrm{H}^{\mathrm{N}}$ distances of 2.6$2.9 \AA$ are seen for all these residues in the crystal structure while in the closed conformation longer distances of $4.6 \AA$ are observed for residues $137-139$. The $\mathrm{H}^{\mathrm{N}}-\mathrm{H}^{\mathrm{N}}$ region of the $3 \mathrm{D}$ NOESY-HSQC spectrum shows significantly weaker NOEs for residues 138-140 than for residues 141-146 (Figure 4). This suggests that the helical secondary structure present at the $\mathrm{N}$ terminus of helix $\alpha 6$ of $\lambda$ lysozyme in solution is more fluctuating in nature. The ${ }^{3} J_{\mathrm{HNH} \alpha}$ coupling constants measured for residues in this region (His137 $6.1 \mathrm{~Hz}$, Lys138 $5.4 \mathrm{~Hz}$, Ser141 $5.9 \mathrm{~Hz}$ ) are also consistent with fluctuating helical secondary structure. Lower coupling constant values of less than $5 \mathrm{~Hz}$, usually considered to be indicative of persistent helical secondary structure, ${ }^{[13]}$ are only seen in helix $\alpha 6$ for residues $144-$ 148. Furthermore, data from $H / D$ exchange studies ${ }^{[12]}$ show that there is protection from exchange for the amides protons of residues 142-149 but not residues 138-141 in helix $\alpha 6$.

\section{${ }^{15} \mathrm{~N}-{ }^{1} \mathrm{H}^{\mathrm{N}}$ residual dipolar couplings}

${ }^{15} \mathrm{~N}-{ }^{1} \mathrm{H}^{\mathrm{N}}$ residual dipolar couplings (RDCs) were measured in $5 \% \mathrm{C} 12 \mathrm{E} 6 / n$-hexanol for 113 residues of $\lambda$ lysozyme. ${ }^{[14]}$ The molecular alignment tensors for the $A$ and $B$ molecules in the $1 \mathrm{AM} 7 \mathrm{X}$-ray structure were fitted to minimize the $\chi^{2}$ between 
the experimental and calculated RDCs for a group of 97 backbone amides with $\left\{{ }^{1} \mathrm{H}\right\}^{15} \mathrm{~N}$ NOE values of greater than 0.75 . Sixteen residues from the $\mathrm{N}$ and $\mathrm{C}$ termini and from the lower and upper lip regions were excluded. Good agreement between the experimental and calculated RDC values is obtained for both the A and B structures with $Q$ values of 0.195 and 0.189 , respectively (Figure 5 ). This indicates that both the $A$ and $B$ structures are good models for the solution structure of $\lambda$ lysozyme in the region of these 97 residues. The fitted alignment tensors were then used to calculate the RDCs expected for all 113 backbone ${ }^{1} \mathrm{H}^{\mathrm{N}}-{ }^{15} \mathrm{~N}$ for which experimental RDCs were measured, including residues from the lower and upper lip regions; $Q$ values of 0.245 and 0.349 were obtained for the A and B structures, respectively. The lower $Q$ value for the A
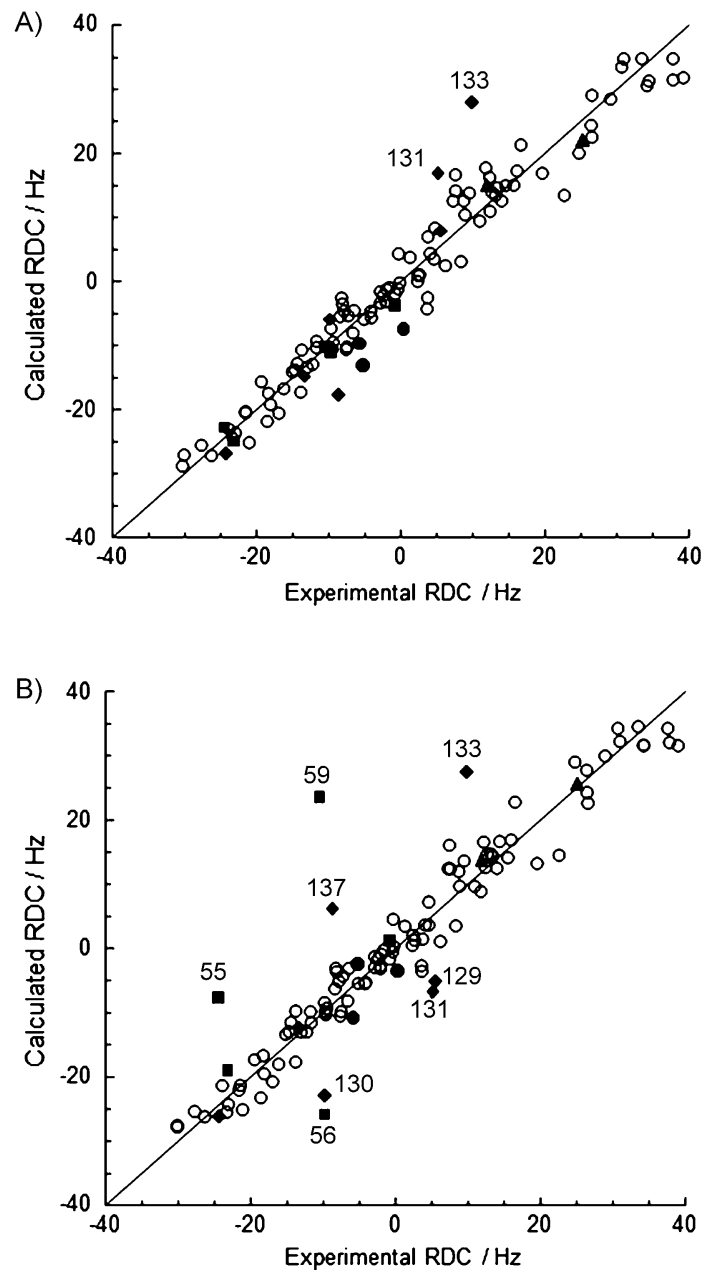

Figure 5. Comparison of experimental RDCs with RDCs calculated from A) molecule A (open form) and B) molecule B (closed form). The 97 residues used for the determination of the alignment tensor are shown as $\bigcirc$. Residues from the $\mathrm{N}$ terminus (3-5), the lower lip $(55,56,58-60)$, the loop between $\alpha 2$ and $\alpha 3(84,85)$ and the upper lip $(129-131,133,137,138,140)$, which were excluded from the fitting of the alignment tensor due to low heteronuclear NOE values, are shown as $\bullet, \mathbf{\square}, \mathbf{\Delta}$ and $\bullet$, respectively. In panel $\mathrm{A}$, where a good agreement is observed overall between the calculated and experimental RDC values, the effects of dynamics may be seen for some residues with lower ${ }^{1} \mathrm{H},{ }^{15} \mathrm{~N}$ order parameters. For example, for Gly 133 the experimental RDC value $(9.8 \mathrm{~Hz})$ is lower than that calculated from the structure of molecule $A(27.9 \mathrm{~Hz})$, although the difference between the RDC values is larger than would be expected from the order parameter $\left(S^{2} 0.82\right)$. structure indicates that this structure provides a better overall model of the solution structure than the $B$ structure. For the $B$ structure particularly large differences between the experimental and calculated RDCs are found for residues 55, 56 and 59 of the lower lip and residues 129-131, 133 and 137 of the upper lip; these residues are highlighted in Figure 5 B. For the A structure much better agreement is obtained for 55, 59, 129, 130 and 137; however, significant deviations are observed for 131 and 133 (Figure $5 \mathrm{~A}$ ).

\section{Molecular dynamics simulations}

Two simulations of $\lambda$ lysozyme have been run at $300 \mathrm{~K}$ starting from molecule $A$ (open form) and molecule B (closed form) in the $1 \mathrm{AM} 7$ crystal structure. ${ }^{[4]}$ Figure 6 shows the root-meansquare fluctuations (RMSF) of the backbone nitrogen atoms of $\lambda$ lysozyme throughout the simulations. Low RMSF values are

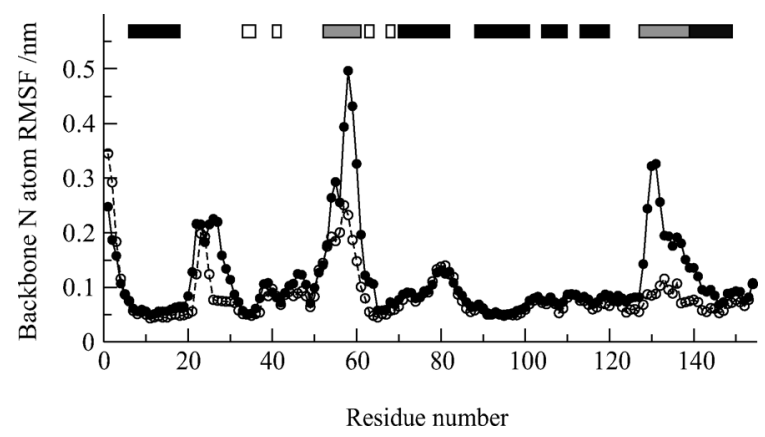

Figure 6. The root-mean-square fluctuations (RMSF) of the backbone nitrogen atoms in the simulations of molecules A (filled symbol and solid line) and $B$ (open symbol and dashed line). The secondary structure present in the $X$-ray structure of molecule B is shown with filled black bars for $\alpha$-helices and open bars for $\beta$-strands. Grey bars indicate the positions of the upper and lower lip region.

seen for residues in the main regions of secondary structure, reflecting the fact that overall the $\lambda$ lysozyme structure is very stable in the GROMOS force field. However, elevated RMSF values greater than $0.15 \mathrm{~nm}$ are seen for residues $1-3,22-28$, 53-61 and 129-138 in the simulation of molecule $A$, and for residues $1-3,23-24$ and $53-59$ in the simulation of molecule $B$. The lower fluctuations seen for residues $129-138$ in the simulation of molecule $B$, compared to those seen for this region in the simulation of molecule $A$, probably reflect the relatively short length (10 ns) of the simulations analysed here. Residues $1-3$ are at the protein $\mathrm{N}$ terminus and residues $22-28$ are in a loop between helix $\alpha 1$ and $\beta$-strand $\beta 1$. Residues $53-61$ and 129-138 are in the lower and upper lip regions, respectively, whose conformation and orientation differ significantly between the open and closed forms. These two regions, which show particularly high RMSF values in the simulation of molecule $\mathrm{A}$, also show low experimental ${ }^{1} \mathrm{H}_{1}^{15} \mathrm{~N}$ order parameters (Figure 2C). Low order parameters are also seen for residues 3 , 26 and 28 which show elevated RMSF values.

To understand the conformational changes involved in giving the elevated RMSF values in the simulations the variations in secondary structure of the protein through the simulations have been analysed (Figure 7). In general, the secondary 


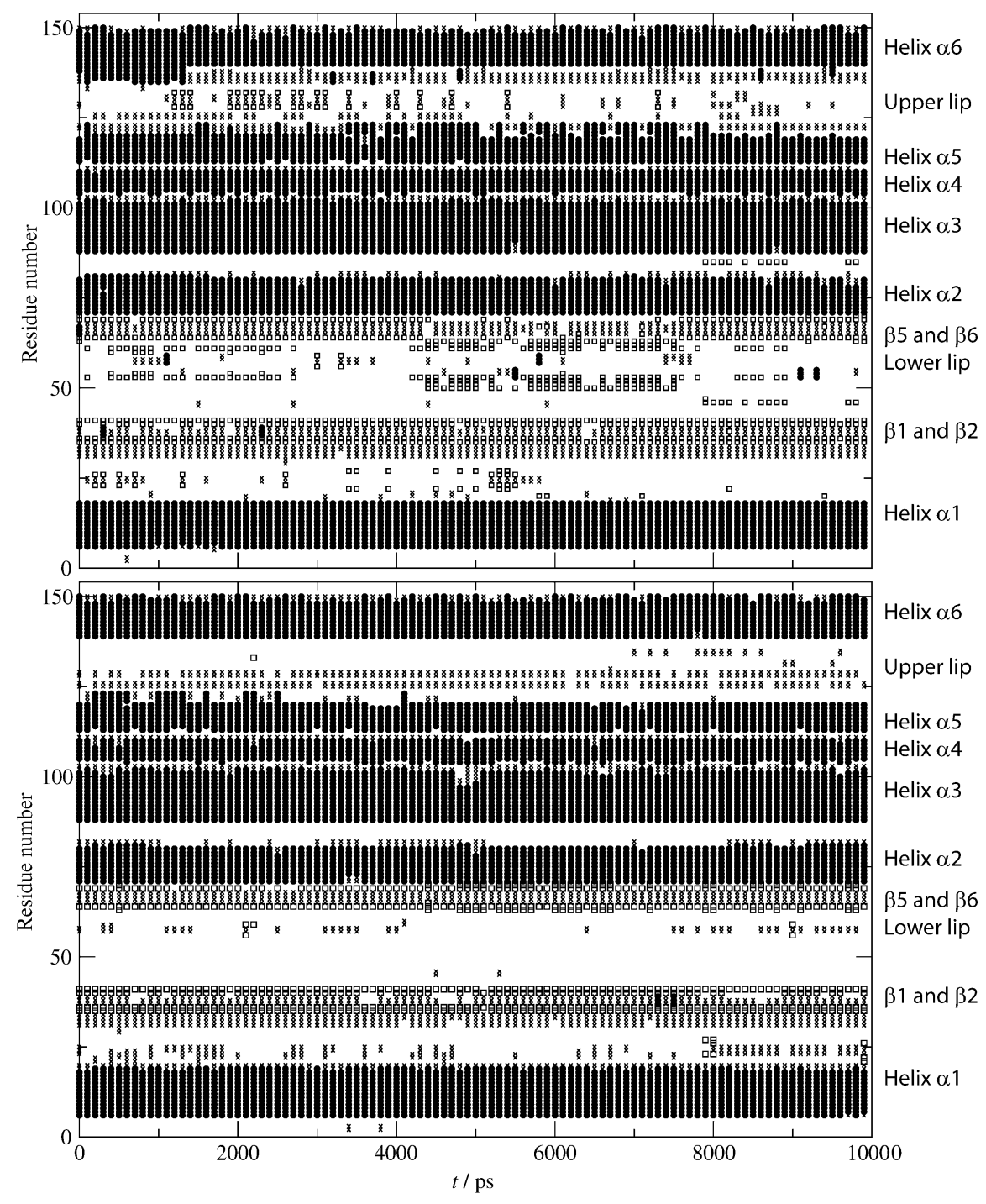

Figure 7. The secondary structure present through the simulations of molecule $A$ (upper panel) and molecule $B$ (lower panel) of $\lambda$ lysozyme. The secondary structure is identified according to the DSSP algorithm ${ }^{[37]}$ and is shown with the following symbols: filled black circle $\alpha$ and $3_{10}$ helix; open square box $\beta$-strand; $x$ symbol turn. distances between residues in the lip region and those in the active-site cleft through the simulations. Examples are shown in Figure 8, which gives the variations in the distance from Lys58 $C^{\alpha}$ in the lower lip region, and Tyr132 $C^{\alpha}$ in the upper lip region, to Glu19 $C^{\alpha}$ in the active-site cleft through the two simulations. As shown in the upper panel of Figure 8 the distance between Lys58 $C^{\alpha}$ in the lower lip region and Glu19 $\mathrm{C}^{\alpha}$ in the active-site cleft varies from 0.82 to $2.29 \mathrm{~nm}$ through the simulations, with an overall reduction in the distance being observed through the simulation of molecule A. In the simulations, therefore, the lower lip region is adopting a dynamic ensemble of conformers, some of which show the $\beta$-sheet interactions seen for strands $\beta 3$ and $\beta 4$ in the $X$-ray structure of molecule A. Such behaviour is consistent with the NMR data, where the chemical shift data are indicative of $\beta$-sheet conformations but no hydrogen-exchange protection is observed. The significant fluctuations seen in the lower lip region during the simulations may play an important role in allowing the substrate to readily access the active-site cleft. structure in $\lambda$ lysozyme is very persistent in the simulations. However, some fluctuations are seen in the lower and upper lip regions, particularly in the simulation starting from molecule $A$ (Figure 7 ). In the lower lip region the $X$-ray structure of molecule $A$ contains $\beta$-strands $\beta 3$ (residues $53-56$ ) and $\beta 4$ (residues 59-61) but these two $\beta$-strands are missing in molecule $B$. In the simulation of molecule $A$ the secondary structure in the lower lip region fluctuates throughout the simulation with some $\beta$-sheet interactions present. In particular the hydrogen bonds $53 \mathrm{NH} \cdots 61 \mathrm{CO}, 61 \mathrm{NH} \cdots 53 \mathrm{CO}$ and $59 \mathrm{NH} \cdots 56 \mathrm{CO}$ have populations of $45.1 \%, 47.8 \%$ and $10.3 \%$, respectively, through the simulation. In the simulation of molecule B the lower lip region mostly adopts unstructured bends. However, the hydrogen bond $59 \mathrm{NH}$...56 CO, which would correspond to a $\beta$-sheet interaction between strands $\beta 3$ and $\beta 4$, has a population of $27.9 \%$.

During the simulations the overall position of the lower lip region fluctuates. To characterise the dynamics of the lip regions in more detail, we have monitored the changes in the
In the X-ray structure, helix $\alpha 6$ in molecule $B$ comprises residues 139-149, whereas in molecule $A$ it runs from residues 135-149 with the $\mathrm{N}$-terminal region of the helix extending into the upper lip region. Within the first nanosecond of the simulation of molecule $\mathrm{A}$ this $\mathrm{N}$-terminal region of helix $\alpha 6$ is lost. However, analysis of the hydrogen bonds during the simulation shows that although the helix is lost, some persistent helical turns remain for residues 135-139 (Figure 7). In particular, the hydrogen bonds $138 \mathrm{NH} \cdots 134 \mathrm{CO}$ and $138 \mathrm{NH} \cdots 135 \mathrm{CO}$ have populations of $58.6 \%$ and $15.2 \%$, respectively, through the simulation. These hydrogen bonds are not seen in the simulation starting from molecule $B$, but the helical hydrogen bond $142 \mathrm{NH} \cdots 138 \mathrm{CO}$ does have a population of $59.9 \%$. As shown in the lower panel of Figure 8 the distance between Tyr132 $C^{\alpha}$ in the upper lip region and Glu19 $C^{\alpha}$ shows an initial drop in the simulation of molecule A during the first nanosecond when the $\mathrm{N}$-terminal region of helix $\alpha 6$ is lost. After that there are only small fluctuations in this distance through both simulations. Indeed, after helix $\alpha 6$ is lost the conformation of 

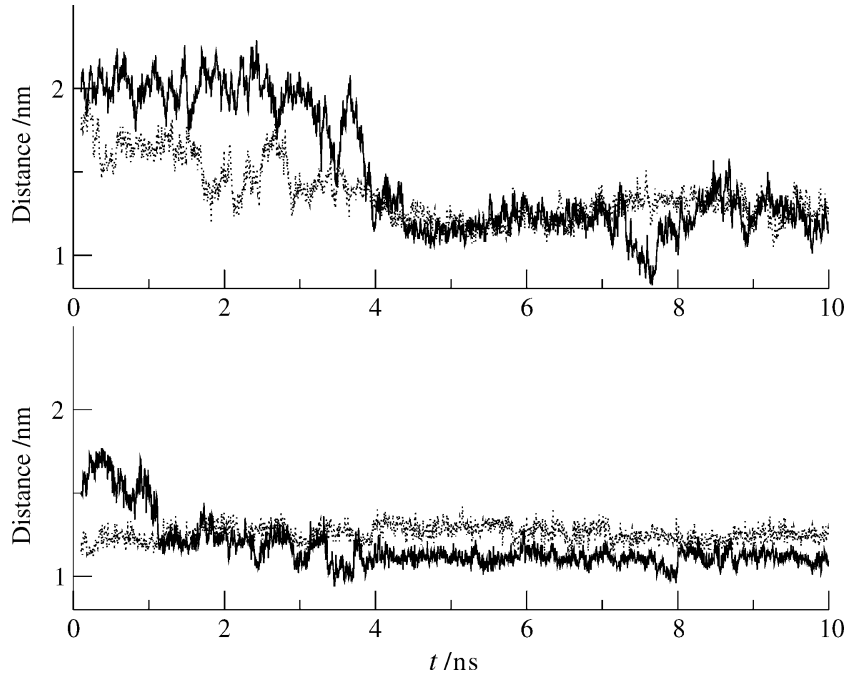

Figure 8. Variations in the distance between the $C^{\alpha}$ atoms of Lys58 in the lower lip and Glu19 in the active site cleft (upper panel) and between the $\mathrm{C}^{\alpha}$ atoms of Tyr132 in the upper lip and Glu19 in the active site cleft (lower panel) through the simulations of $\lambda$ lysozyme. The data for the simulation starting from molecule $A$ is shown in a solid line and the data for the simulation starting from molecule $B$ is shown in a dotted line.

the upper lip is quite similar in both the simulations, its overall orientation resembling that seen in molecule $B$ in the $X$-ray structure (Figure 1B). However, in contrast to molecule $B$ in the $X$-ray structure, the upper lip region contains fluctuating helical secondary structure at the $\mathrm{N}$ terminus of helix $\alpha 6$ in the simulations. This is in agreement with the experimental chemical shift, NOE and hydrogen exchange data.

\section{Conclusions}

Two different conformations for the upper and lower lip regions of $\lambda$ lysozyme could be identified in X-ray structures. ${ }^{[4]}$ In the open form, the lower lip region contains two $\beta$-strands ( $\beta 3$ and $\beta 4$ ) and the initial helical turn of helix $\alpha 6$ is present in the upper lip region. In the closed form, these regions of secondary structure are lost and the loop regions extend across the active-site cleft. The experimental chemical shift, ${ }^{[9]} \mathrm{RDC},{ }^{3} \mathrm{JNH}_{\mathrm{HN}}$ and NOE data suggest that both the $\beta$-strands in the lower lip and the initial helical region at the start of helix $\alpha 6$, seen in the open form, are retained in solution. However, the ${ }^{15} \mathrm{~N}$ relaxation and hydrogen exchange ${ }^{[12]}$ data show that these regions display a significant degree of mobility in solution. This conclusion is supported by the MD simulations, which show fluctuating $\beta$-sheet hydrogen bonds in the lower lip region and dynamic helical turns in the $\mathrm{N}$-terminal region of helix $\alpha 6$ in the upper lip region. Interestingly, in the $1 \mathrm{AM} 7$ crystal structure there are hydrogen bonds between Pro57 and Lys58 in molecule B and Asn6 in molecule C. Residues 57 and 58 are at the start of $\beta$-strand $\beta 4$ which is missing in molecule $B$. It may be therefore that crystal contacts are helping to stabilise the conformation without the $\beta$-sheet hydrogen bonds in the lower lip region in the closed conformation. No intermolecular hydrogen bonds are seen for residues 57 and 58 in molecules $A$ or $\mathrm{C}$ which are in the open form in the crystal structure.
On the basis of crystallographic data, combined with studies in which His137 in $\lambda$ lysozyme was chemically modified or mutated, Evrard et al. identified that both the open and closed forms of $\lambda$ lysozyme may have a functional role. ${ }^{[15]}$ They proposed that the open form is required to allow the peptidoglycan to access the active-site cleft while the closed form is needed for enzyme activity. In particular, chemical modification of His137 in the upper lip by N-carbethoxylation, which may prevent $\lambda$ lysozyme from adopting the closed conformation, was shown to make the enzyme inactive. ${ }^{[15]}$ In agreement with this, the structure of $\lambda$ lysozyme in complex with hexa- $N$-acetylchitohexaose (PDB ID: 3D3D ${ }^{[4 b]}$ ) has a close similarity to the structure of molecule $B$ (closed conformation) in the $1 \mathrm{AM} 7$ crystal structure. The data reported here show that in solution the upper and lower lip regions adopt an ensemble of different conformations, rather than just the open and closed forms. The dynamic nature of the upper and lower lips should enable entry of the substrate into the active site and this can then be followed by population of the conformations within the ensemble that give enzyme activity.

\section{Experimental Section and Computational Methods}

${ }^{15} \mathrm{~N}$ NMR relaxation experiments: ${ }^{15} \mathrm{~N}$-labelled $\lambda$ lysozyme was expressed and purified as described previously. ${ }^{[9]}$ NMR relaxation experiments were performed at a $\lambda$ lysozyme concentration of $\sim 0.5 \mathrm{~mm}$ in $95 \% \mathrm{H}_{2} \mathrm{O} / 5 \% \mathrm{D}_{2} \mathrm{O}$ at $\mathrm{pH} 5.45$ and $20^{\circ} \mathrm{C}$ on home-built spectrometers equipped with triple-resonance probes with ${ }^{1} \mathrm{H}$ operating frequencies of 500.10 and $750.04 \mathrm{MHz}$. Pulse sequences for the measurement of the longitudinal $\left(T_{1}\right)$ and transverse $\left(T_{2}\right)$ relaxation times and the $\left\{{ }^{1} \mathrm{H}\right\}^{15} \mathrm{~N}$ heteronuclear NOE of the backbone ${ }^{15} \mathrm{~N}$ nuclei have been described previously. ${ }^{[16]} T_{1}$ and $T_{2}$ measurements were performed using series of eleven experiments with relaxation delays ranging from 20 to $2000 \mathrm{~ms}$, for $T_{1}$, and from 8 to $355 \mathrm{~ms}$, for $T_{2}$. The Carr-Purcell-Meiboom-Gill delay $\left(2 \tau_{\mathrm{cp}}\right)$ for the $T_{2}$ experiments was $1 \mathrm{~ms}$. A recycle delay of $2 \mathrm{~s}$ was used for all $T_{1}$ and $T_{2}$ experiments. The $\left\{{ }^{1} \mathrm{H}\right\}^{15} \mathrm{~N}$ NOE experiments were recorded with and without ${ }^{1} \mathrm{H}$ saturation for $3 \mathrm{~s}$ at $500 \mathrm{MHz}$ and $4 \mathrm{~s}$ at $750 \mathrm{MHz}$. The data sets were acquired using 100 and 128 complex $t_{1}$ increments with ${ }^{15} \mathrm{~N}$ sweep widths of 1515.15 and $2272.73 \mathrm{~Hz}$ at ${ }^{15} \mathrm{~N}$ frequencies of 50.68 and $76.01 \mathrm{MHz}$, respectively. $1 \mathrm{~K}$ complex data points were recorded in the $F_{2}$ dimension with sweep widths of 7142.86 and $10526.32 \mathrm{~Hz}$ at 500 and $750 \mathrm{MHz}$, respectively. 24 scans were collected per $t_{1}$ increment for $T_{1}$ and $T_{2}$ measurements at $500 \mathrm{MHz}$ and 16 scans at $750 \mathrm{MHz} .112$ and 160 scans were collected for the $\left\{{ }^{1} \mathrm{H}\right\}^{15} \mathrm{~N}$ NOE experiments at 500 and $750 \mathrm{MHz}$, respectively.

${ }^{15} \mathrm{~N}$ relaxation data were collected for 128 of the 158 residues of $\lambda$ lysozyme. Data for the remaining residues could not be measured due to weak or overlapping peaks. $T_{1}$ and $T_{2}$ were fitted as single-exponential decays to the peak intensities determined as a function of the eleven delay times. The $\left\{{ }^{1} \mathrm{H}\right\}^{15} \mathrm{~N}$ NOE was calculated as the ratio of the peak intensities in the spectra recorded with and without ${ }^{1} \mathrm{H}$ saturation. Peak heights were determined using inhouse peak-picking software. Uncertainties in the $T_{1}, T_{2}$ and $\left\{{ }^{1} \mathrm{H}\right\}^{15} \mathrm{~N}$ NOE values were estimated from 500 Monte Carlo simulations using the baseline noise as a measure of the error in the peak heights. The ${ }^{15} \mathrm{~N} T_{1}, T_{2}$ and $\left\{{ }^{1} \mathrm{H}\right\}^{15} \mathrm{~N}$ NOE values at 500 and $750 \mathrm{MHz}$ have been deposited in the BMRB with accession number 19127. 
The relaxation data for $\lambda$ lysozyme were analysed with an axially symmetric rotational diffusion tensor. ${ }^{[17]}$ This tensor was determined by comparison of experimental $T_{1} / T_{2}$ ratios with those calculated using the Lipari-Szabo formalism ${ }^{[8]}$ with spectral density functions appropriate for axially symmetric rotational diffusion. ${ }^{[18]}$ The magnitudes $\left(D_{\|}, D_{\perp}\right)$ and orientation $(\theta, \phi)$ of the principle components of the rotational diffusion tensor were searched to optimize the agreement between experimental and calculated $T_{1} / T_{2}$ ratios using the $1 \mathrm{AM} 7 \mathrm{X}$-ray structure. ${ }^{[4]]}$ Calculations of the $T_{1} / T_{2}$ ratio were carried out with a fixed $S^{2}$ value of 0.9 , an $\mathrm{N}-\mathrm{H}$ bond length of $1.02 \AA$ and $a{ }^{15} \mathrm{~N}$ chemical shift anisotropy, $\left(\sigma_{\|}-\sigma_{\perp}\right)$, of $-170 \mathrm{ppm}$. The principal component of the ${ }^{15} \mathrm{~N}$ chemical shift tensor was assumed to lie in the peptide plane and to form an angle of $19^{\circ}$ with the $\mathrm{N}-\mathrm{H}$ bond vector. ${ }^{[19]}$ Residues for which the $T_{1} / T_{2}$ ratios are affected by fast or slow molecular motions were excluded from this analysis. The diffusion tensor used in the modelfree analysis described below was determined using $T_{1} / T_{2}$ ratios for 85 residues. Analysis of the $T_{1} / T_{2}$ ratios at 500 and $750 \mathrm{MHz}$ resulted in slightly different overall rotational correlation times, $\tau_{\mathrm{R}}=1 /$ $2\left(D_{\|}+2 D_{\perp}\right)$, of 10.8 and $11.3 \mathrm{~ns}$, respectively. These differences are likely to have arisen due to slightly different sample temperature in the two spectrometers. In order to correct for this, a scaling factor for each field was used in the simultaneous fitting of the diffusion tensor to the $T_{1} / T_{2}$ data collected at the two fields; a final value of 1.278 was obtained for the $D_{\|} / D_{\perp}$ ratio.

Relaxation data were analysed using in-house software; this incorporates the model-free formalism of Lipari and Szabo, ${ }^{[8]}$ using spectral density functions appropriate for axially symmetric rotational diffusion $^{[18]}$ and non-colinearity of the $\mathrm{N}-\mathrm{H}$ bond vector and the principal component of the ${ }^{15} \mathrm{~N}$ chemical shift tensor, ${ }^{[19 a]}$ with model selection and Monte Carlo error estimation as described by Mandel et al. ${ }^{[20]}$ The relaxation data were fitted using six models: $S^{2}$ only (model 1$), S^{2}$ and $R_{\text {ex }}$ (model 2), $S^{2}$ and $\tau_{\mathrm{e}}$ (model 3 ), $S^{2}, R_{\mathrm{ex}}$ and $\tau_{\mathrm{e}}$ (model 4), the extended model of Clore et al. ${ }^{[21]}$ with $S^{2}{ }_{f}, S^{2}{ }_{\mathrm{s}}$ and $\tau_{\mathrm{s}}$ (model 5), and the extended model with $S^{2}{ }_{\mathrm{f}}, S_{\mathrm{s}^{\prime}}^{2} \tau_{\mathrm{s}}$ and $R_{\mathrm{ex}}$ (model 6). $S^{2}$ is the generalized order parameter, $R_{\mathrm{ex}}$ is the chemical exchange contribution to $T_{2}$, and $\tau_{\mathrm{e}}$ is the effective correlation time for internal motion on a fast timescale. $S_{{ }_{f}}^{2} S_{{ }_{s}}^{2}$ and $\tau_{s}$, used in models 5 and 6 , are the order parameters for faster and slower timescale motions and the effective correlation time for slow internal motion, respectively; $\tau_{\mathrm{f}}$, the internal correlation time for fast motion in the extended model, was assumed to be sufficiently small and, therefore, not to contribute significantly to the relaxation parameters. ${ }^{[21]}$ In models 2,4 and 6 , a single $R_{\text {ex }}$ term was used to fit the two $T_{2}$ values for each residue and this $R_{\text {ex }}$ term was scaled with the square of the field strength. ${ }^{[22]}$ Factors were introduced in the spectral density functions to scale the values of $D_{\|}$ and $D_{\perp}$ to take into account the small deviations between data sets recorded at 500 and $750 \mathrm{MHz}$ due to temperature variation. For each model, best fits for $S^{2}$, and other relevant parameters $\left(R_{\mathrm{ex}}\right.$ $\tau_{\mathrm{e}}, S^{2}{ }_{\mathrm{f}} S_{{ }^{\prime}}^{2}$ and $\tau_{\mathrm{s}}$ ) were determined by minimizing the $\chi^{2}$ parameter using a downhill simplex algorithm. ${ }^{[23]}$ The error in these parameters was estimated from 500 Monte Carlo simulations (as one standard deviation from the mean value). The statistical significance of an improvement in $\chi^{2}$ when a model with additional parameters is used was assessed with an F-statistic. A more complex model was considered to be statistically significant if a $p$-value of less than 0.2 was obtained from the F-statistic. ${ }^{[20]}$

Measurement of chemical shift, coupling constant and NOE data: Backbone ${ }^{1} \mathrm{H},{ }^{13} \mathrm{C}$ and ${ }^{15} \mathrm{~N}$ assignments for $\lambda$ lysozyme have been published. ${ }^{[9]}{ }^{3} \mathrm{HNH}_{\mathrm{HNa}}$ coupling constants were measured using a 2D HMQC-J $\mathrm{J}^{[24]}$ dataset collected at $750 \mathrm{MHz}$. The sweep widths were 10526.32 and $2272.73 \mathrm{~Hz}$ in $F_{2}$ and $F_{1}$, and 1024 and 375 complex points were collected in $t_{2}$ and $t_{1}$, respectively. After zerofilling the digital resolution in $F_{1}\left({ }^{15} \mathrm{~N}\right)$ was $0.55 \mathrm{~Hz} / \mathrm{pt}$. The coupling constants were extracted from $F_{1}$ columns using an in-house fitting program; the ${ }^{3} J_{\mathrm{HNH} \alpha}$ values have been deposited in the BMRB with accession number 19127. ${ }^{1} \mathrm{H}-{ }^{1} \mathrm{H}$ NOEs were obtained from a 3D ${ }^{15} \mathrm{~N}$-edited NOESY-HSQC spectrum collected at $750 \mathrm{MHz}$ with a mixing time of $150 \mathrm{~ms}^{\left[{ }^{[9]}\right.}$

Residual dipolar couplings: Liquid crystals from $n$-alkyl-poly(ethylene glycols) and $n$-alkyl alcohols were prepared as described by Rückert and Otting. ${ }^{[14]}$ To prepare a stock solution, $n$-hexanol $(18 \mu \mathrm{L})$ was added to a $15 \%$ C12E6 solution (500 $\mu \mathrm{L})$. The sample tube was shaken. When the two compounds had mixed sufficiently the solution was inspected for a bluish tinge indicative of the formation of the liquid-crystalline phase.

The isotropic data were collected first by using a sample of $\lambda$ lysozyme $(275 \mu \mathrm{L})$ at $\mathrm{pH} 5.45$ and $20^{\circ} \mathrm{C}$ using a Shigemi microcell. Following the isotropic measurement, the $\lambda$ lysozyme solution $(190 \mu \mathrm{L})$ was combined with the $15 \% \mathrm{C} 12 \mathrm{E} 6 / n$-hexanol stock solution $(95 \mu \mathrm{L})$ resulting in a final $\mathrm{C} 12 \mathrm{E} 6 / n$-hexanol concentration of $5 \%$. The sample tube was shaken and again inspected for the bluish tinge; a further $0.5 \mu \mathrm{L}$ of $n$-hexanol was added to achieve this.

NMR experiments were performed on a home-built NMR spectrometer operating at a ${ }^{1} \mathrm{H}$ frequency of $750 \mathrm{MHz} .{ }^{15} \mathrm{~N}-{ }^{1} \mathrm{H}^{\mathrm{N}} \mathrm{RDCs}$ were measured at $20^{\circ} \mathrm{C}$ using the in-phase/antiphase (IPAP) HSQC experiment. ${ }^{[25]} 128$ and 1024 complex points and sweep widths of 2500.0 and $10526.32 \mathrm{~Hz}$ were collected in $F_{1}\left({ }^{15} \mathrm{~N}\right)$ and $F_{2}\left({ }^{1} \mathrm{H}\right)$, respectively. The data were processed using NMRPipe ${ }^{[26]}$ and zerofilled to give final digital resolutions of $1.2 \mathrm{~Hz} / \mathrm{pt}$ and $5.1 \mathrm{~Hz} / \mathrm{pt}$ in $F_{1}$ and $F_{2}$, respectively. Residual dipolar couplings were measured as the difference between the splitting observed in the isotropic and aligned data sets; these RDCs have been deposited in the BMRB with accession number 19127.

The principle components $\left(A_{x x}, A_{y y}\right.$ and $\left.A_{z z}\right)$ and orientation $(\phi, \theta$ and $\psi$ ) of the molecular alignment tensor for the $\mathrm{A}$ and $\mathrm{B}$ molecules in the 1AM7 X-ray structure were fitted to minimize the $\chi^{2}$ value between the experimental and calculated RDCs for a group of 97 backbone amides. Residues with $\left\{{ }^{1} \mathrm{H}\right\}^{15} \mathrm{~N}$ heteronuclear NOE values of less than 0.75 were excluded from the fitting procedure. The fitted values of the principle components and orientation of the alignment tensor were then used to calculate the RDCs expected for all 113 backbone ${ }^{1} \mathrm{H}^{\mathrm{N}}-{ }^{15} \mathrm{~N}$ for which experimental RDCs were measured. The quality of the fits were assessed with $Q$, the quality

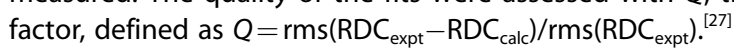

Analysis of the crystal structures: ${ }^{1} \mathrm{H},{ }^{13} \mathrm{C}$, and ${ }^{15} \mathrm{~N}$ chemical shifts for $\lambda$ lysozyme were predicted using the SHIFTX2 $2^{[10]}$ program for molecules A and B in the 1AM7 crystal structure ${ }^{[4 a]}$ (aza-tryptophan residues in the crystal structure were converted to tryptophan for the prediction). TALOS $+{ }^{[11]}$ was used to predict the $\phi, \psi$ torsion angle values from the experimental chemical shift data. ${ }^{[9]}{ }^{3} J_{\mathrm{HNHa}}$ coupling constant values were predicted from the $\phi$ torsion angles in molecules $A$ and $B$ in the $1 A M 7$ crystal structure ${ }^{[4 a]}$ using the Karplus equation coefficients of Pardi et al. ${ }^{[28]}$

Crystal contacts between the different molecules within the 1AM7 crystal structure were analysed using the protein interfaces, surfaces and assemblies (PISA) service at the European Bioinformatics Institute (http://www.ebi.ac.uk/pdbe/prot_int/pistart.html). ${ }^{[29]}$

Molecular dynamics simulations: Simulations of $\lambda$ lysozyme were performed using the GROMOS biomolecular simulation software ${ }^{[30]}$ 
and the 45A3 GROMOS force field. ${ }^{[31]}$ Initial coordinates of the protein were taken from the crystal structure of $\lambda$ lysozyme with PDB ID: $1 A M 7$ using molecules $A$ and $B{ }^{[4 a]}$ All the Trp residues in $\lambda$ lysozyme had been replaced by aza-tryptophan in the experimental structure determination. These aza-tryptophans were converted to Trp residues for the simulations. All the Asp and Glu side chains in the protein were unprotonated and the three His side chains were singly protonated in the simulation. The protein was solvated in a truncated octahedral box containing 5063 (simulation of molecule A) and 5064 (simulation of molecule B) simple point charge (SPC) water molecules. ${ }^{[32]}$

The simulations were performed at a temperature of $300 \mathrm{~K}$, following initial runs with the solute atoms restrained to their positions in the X-ray structure of $2 \mathrm{ps}$ at $100 \mathrm{~K}$ and $2 \mathrm{ps}$ at $200 \mathrm{~K}$. Two $10 \mathrm{~ns}$ simulations were performed, one starting from molecule $A$ and the other from molecule B. All the simulations were performed at constant pressure $(1 \mathrm{~atm})$, the temperature and pressure being maintained by weak coupling to an external bath ${ }^{[33]}$ (temperature coupling relaxation time $0.1 \mathrm{ps}$; pressure coupling relaxation time $0.5 \mathrm{ps}$; isothermal compressibility $\left.k_{\mathrm{T}} 4.575 \times 10^{-4}\left(\mathrm{KJ} \mathrm{mol}^{-1} \mathrm{~nm}^{-3}\right)^{-1}\right)$. Throughout the simulations bond lengths were constrained to ideal values using the SHAKE procedure with a geometric accuracy of $10^{-4} \cdot{ }^{[34]}$ Nonbonded interactions were treated using a twin range method. ${ }^{[35]}$ Within a short-range cutoff of $0.8 \mathrm{~nm}$ all interactions were determined at every step. Longer range (electrostatic and van der Waals) interactions within a cutoff range of $1.4 \mathrm{~nm}$ were updated at the same time as the pair list was generated (every $10 \mathrm{fs}$ ). A reaction field was applied $\left(\begin{array}{ll}\varepsilon & 61.0^{[36]}\end{array}\right)$ beyond a cutoff of $1.4 \mathrm{~nm}$. A time step of $2 \mathrm{fs}$ was used, analysis being performed using trajectory coordinates and energies written to disk every $0.1 \mathrm{ps}$.

Regions of secondary structure within the simulation conformers were identified using the program DSSP. ${ }^{[37]}$ Hydrogen bonds are defined geometrically: a hydrogen bond is assumed to exist if the hydrogen-acceptor distance is smaller than $0.25 \mathrm{~nm}$ and the donor-hydrogen-acceptor angle is larger than $135^{\circ} .^{[30 a]}$

\section{Acknowledgements}

We thank the Oxford Supercomputing Centre for access to their facilities. A.M. was supported in part by the Fonds de la Recherche Fondamentale Collective (FRFC, grant number 2.4530.09) and A.D.P. was the recipient of a F.R.I.A. PhD fellowhip. A.M. and C.R. acknowledge support from the Belgian program of Interuniversity Attraction Poles, initiated by the Federal Office for Scientific Technical and Cultural Affaires (PAI $n^{\circ}$ P7/44).

Keywords: ${ }^{15} \mathrm{~N}$ relaxation - lysozymes - molecular dynamics NMR spectroscopy $\cdot$ residual dipolar couplings

[1] a) L. W. Black, D. S. Hogness, J. Biol. Chem. 1969, 244, 1982-1987; b) M. Imada, A. Tsugita, Nat. New Biol. 1971, 233, 230.

[2] a) A. Taylor, B. C. Das, J. Vanheijenoort, Eur. J. Biochem. 1975, 53, 47-54 b) K. Bieńkowska-Szewczyk, B. Lipińska, A. Taylor, Mol. Gen. Genet. 1981, $184,111-114$.

[3] K. E. van Straaten, T. R. M. Barends, B. W. Dijkstra, A.-M. W. H. Thunnissen, J. Biol. Chem. 2007, 282, 21197-21205.

[4] a) C. Evrard, J. Fastrez, J. P. Declercq, J. Mol. Biol. 1998, 276, 151-164; b) A. K. W. Leung, H. S. Duewel, J. F. Honek, A. M. Berghuis, Biochemistry 2001, 40, 5665-5673.
[5] L. Jespers, E. Sonveaux, J. Fastrez, J. Mol. Biol. 1992, 228, 529-538.

[6] A. L. Lovering, L. H. de Castro, D. Lim, N. C. J. Strynadka, Science 2007, 315, 1402-1405.

[7] Y. Yuan, D. Barrett, Y. Zhang, D. Kahne, P. Sliz, S. Walker, Proc. Natl. Acad. Sci. USA 2007, 104, 5348-5353.

[8] a) G. Lipari, A. Szabo, J. Am. Chem. Soc. 1982, 104, 4546-4559; b) G. Lipari, A. Szabo, J. Am. Chem. Soc. 1982, 104, 4559-4570.

[9] A. Di Paolo, V. Duval, A. Matagne, C. Redfield, Biomol. NMR Assignments 2010, 4, $111-114$

[10] B. Han, Y. Liu, S. W. Ginzinger, D. S. Wishart, J. Biomol. NMR 2011, 50, 43-57.

[11] Y. Shen, F. Delaglio, G. Cornilescu, A. Bax, J. Biomol. NMR 2009, 44, $213-$ 223.

[12] A. Di Paolo, D. Balbeur, E. De Pauw, C. Redfield, A. Matagne, Biochemistry 2010, 49, 8646-8657.

[13] L. J. Smith, K. A. Bolin, H. Schwalbe, M. W. MacArthur, J. M. Thornton, C. M. Dobson, J. Mol. Biol. 1996, 255, 494-506.

[14] M. Rückert, G. Otting, J. Am. Chem. Soc. 2000, 122, 7793-7797.

[15] C. Evrard, J. Fastrez, P. Soumillion, FEBS Lett. 1999, 460, $442-446$.

[16] a) J. Boyd, U. Hommel, I. D. Campbell, Chem. Phys. Lett. 1990, 175, $477-$ 482 ; b) L. E. Kay, D. A. Torchia, A. Bax, Biochemistry 1989, 28, $8972-$ 8979 ; c) A. G. Palmer, N. J. Skelton, W. J. Chazin, P. E. Wright, M. Rance, Mol. Phys. 1992, 75, 699-711.

[17] N. Tjandra, S. E. Feller, R. W. Pastor, A. Bax, J. Am. Chem. Soc. 1995, 117, $12562-12566$.

[18] A. Abragam in The Principles of Nuclear Magnetism, Clarendon, Oxford, 1961.

[19] a) J. Boyd, C. Redfield, J. Am. Chem. Soc. 1998, 120, 9692-9693; b) J. Boyd, C. Redfield, J. Am. Chem. Soc. 1999, 121, 7441-7442; c) G. Cornilescu, A. Bax, J. Am. Chem. Soc. 2000, 122, 10143-10154.

[20] A. M. Mandel, M. Akke, A. G. Palmer, J. Mol. Biol. 1995, 246, 144-163.

[21] G. M. Clore, A. Szabo, A. Bax, L. E. Kay, P. C. Driscoll, A. M. Gronenborn, J. Am. Chem. Soc. 1990, 112, 4989-4991.

[22] O. Millet, J. P. Loria, C. D. Kroenke, M. Pons, A. G. Palmer, J. Am. Chem. Soc. 2000, 122, 2867-2877.

[23] M. L. Johnson, L. M. Faunt, Methods Enzymol. 1992, 210, 1- 37.

[24] L. E. Kay, A. Bax, J. Magn. Reson. 1990, 86, 110-126.

[25] M. Ottiger, F. Delaglio, A. Bax, J. Magn. Reson. 1998, 131, 373-378.

[26] F. Delaglio, S. Grzesiek, G. W. Vuister, G. Zhu, J. Pfeifer, A. Bax, J. Biomol. NMR 1995, 6, 277-293.

[27] G. Cornilescu, J. L. Marquardt, M. Ottiger, A. Bax, J. Am. Chem. Soc. 1998, 120, 6836-6837.

[28] A. Pardi, M. Billeter, K. Wuthrich, J. Mol. Biol. 1984, 180, 741 - 751.

[29] E. Krissinel, K. Henrick, J. Mol. Biol. 2007, 372, 774-797.

[30] a) W. F. van Gunsteren, S. Billeter, A. Eising, P. Hünenberger, P. Krüger, A Mark, W. Scott, I. Tironi, Biomolecular Simulation: The GROMOS96 Manual and User Guide, Vdf Hochschulverlag AG an der ETH Zürich, Zürich, 1996; b) N. Schmid, J. R. Allison, J. Dolenc, A. P. Eichenberger, A.P. E. Kunz, W. F. van Gunsteren, J. Biomol. NMR 2011, 51, 265-281; c) N. Schmid, C. D. Christ, M. Christen, A. P. Eichenberger, W. F. van Gunsteren, Comput. Phys. Commun. 2012, 183, 890-903.

[31] L. D. Schuler, X. Daura, W. F. van Gunsteren, J. Comput. Chem. 2001, 22, $1205-1218$.

[32] H. J. C. Berendsen, J. P. M. Postma, W. F. van Gunsteren, J. Hermans in Intermolecular Forces (Ed.: B. Pullman), Reidel, Dordrecht, 1981, pp. $331-342$

[33] H. J. C. Berendsen, J. P. M. Postma, W. F. van Gunsteren, A. Dinola, J. R. Haak, J. Chem. Phys. 1984, 81, 3684-3690.

[34] J. P. Ryckaert, G. Ciccotti, H. J. C. Berendsen, J. Comput. Phys. 1977, 23, $327-341$.

[35] W. F. van Gunsteren, H. J. C. Berendsen, Angew. Chem. 1990, 102, 1020 1055: Angew. Chem. Int. Ed. Engl. 1990, 29, 992-1023.

[36] T. N. Heinz, W. F. van Gunsteren, P. H. Hunenberger, J. Chem. Phys. 2001, $115,1125-1136$

[37] W. Kabsch, C. Sander, Biopolymers 1983, 22, 2577-2637.

Received: March 28, 2013

Published online on June 25, 2013 